\title{
Management of retrograde type A IMH with acute arch tear/type B dissection
}

\author{
Foeke Nauta ${ }^{1}$, Hector de Beaufort ${ }^{2}$, Firas F. Mussa ${ }^{3}$, Carlo De Vincentiis ${ }^{4}$, Atsushi Omura ${ }^{5}$, \\ Hitoshi Matsuda ${ }^{5}$, Santi Trimarchi ${ }^{6,7}$
}

${ }^{1}$ Department of Cardiothoracic Surgery, Amsterdam UMC, Amsterdam, The Netherlands; ${ }^{2}$ Department of Cardiothoracic Surgery, St Antonius Hospital, Nieuwegein, The Netherlands; ${ }^{3}$ University of South Carolina School of Medicine, Columbia, SC, USA; ${ }^{4}$ Department of Cardiac Surgery, IRCCS Policlinico San Donato, San Donato Milanese, Italy; ${ }^{5}$ Department of Cardiovascular Surgery, National Cerebral and Cardiovascular Center, Suita, Japan; ${ }^{6}$ Department of Surgery, Fondazione IRCCS Cà Granda Ospedale Maggiore Policlinico Milan, Milan, Italy; ${ }^{7}$ Department of Clinical and Community Sciences, University of Milan, Milan, Italy

Correspondence to: Santi Trimarchi, MD, PhD. Department of Surgery, Fondazione IRCCS Cà Granda Ospedale Maggiore Policlinico Milan, Italy; Department of Clinical and Community Sciences, University of Milan, Milan, Italy. Email: santi.trimarchi@.unimi.it.

\begin{abstract}
The incidence of intramural hematomas $(\mathrm{IMH})$ in acute dissection (AD) patients varies between $6 \%$ and $30 \%$ in the literature, most frequently involving only the descending aorta (58\%) than the arch or ascending aorta $(42 \%)$. In this setting, IMH that initiate in the descending aorta, but extend into the arch or ascending aorta have been described, and referred to as a retrograde type A IMH. In these patients the risk of neurological or cardiac complications are high, and therefore an open surgical or hybrid approach has been proposed as the most appropriate. Nevertheless, the endovascular management of such lesions in surgically unfit patients for open surgery have been offered with acceptable outcomes, although the risk of landing in an unsuitable proximal landing zone is evident. In conclusion, retro-TAIMH is an acute aortic syndrome and should be managed as such. The recommended treatment strategy is open surgery for treating ascending or arch involvement, and TEVAR/medical, based on a complication-specific approach, for those with only descending localization. In those patients in whom retro-TAIMH is associated with an acute B dissection presenting with a proximal entry tear located into the descending aorta, a TEVAR represents an option treatment.
\end{abstract}

Keywords: Dissection; intramural hematoma; aorta

Submitted Jul 01, 2019. Accepted for publication Aug 04, 2019.

doi: $10.21037 /$ acs.2019.08.05

View this article at: http://dx.doi.org/10.21037/acs.2019.08.05

\section{Definitions and incidence of aortic intramural hematoma}

An acute aortic intramural hematoma (IMH) is defined by the presence of hemorrhage within the aortic wall without evidence of an intimal tear. This aortic injury may evolve into an aortic dissection (AD) and/or rupture and is then often lethal. It is therefore considered an acute aortic syndrome. The Stanford classifications of $\mathrm{AD}$ are also used for IMH, with type A IMH (TAIMH) defined as involvement of the ascending aorta, and type B IMH (TBIMH) defined as lack of involvement of the ascending aorta. Intramural hematomas that initiate in the descending aorta, but extend into the arch or ascending aorta are referred to as a retrograde type A IMH (retroTAIMH, Figure 1). The incidence of IMH in AD patients varies between $10 \%$ and $30 \%$ in the literature (1-5). In the largest series based on the International Registry of Acute Aortic Dissection (IRAD), the overall incidence of IMH among all acute dissections was 6\% (6). Most IMH patients present with hematoma involving only the descending aorta (58\%), rather than the arch or ascending aorta (42\%). This is contrary to classic $\mathrm{AD}$, which is primarily regarded as type A AD. 

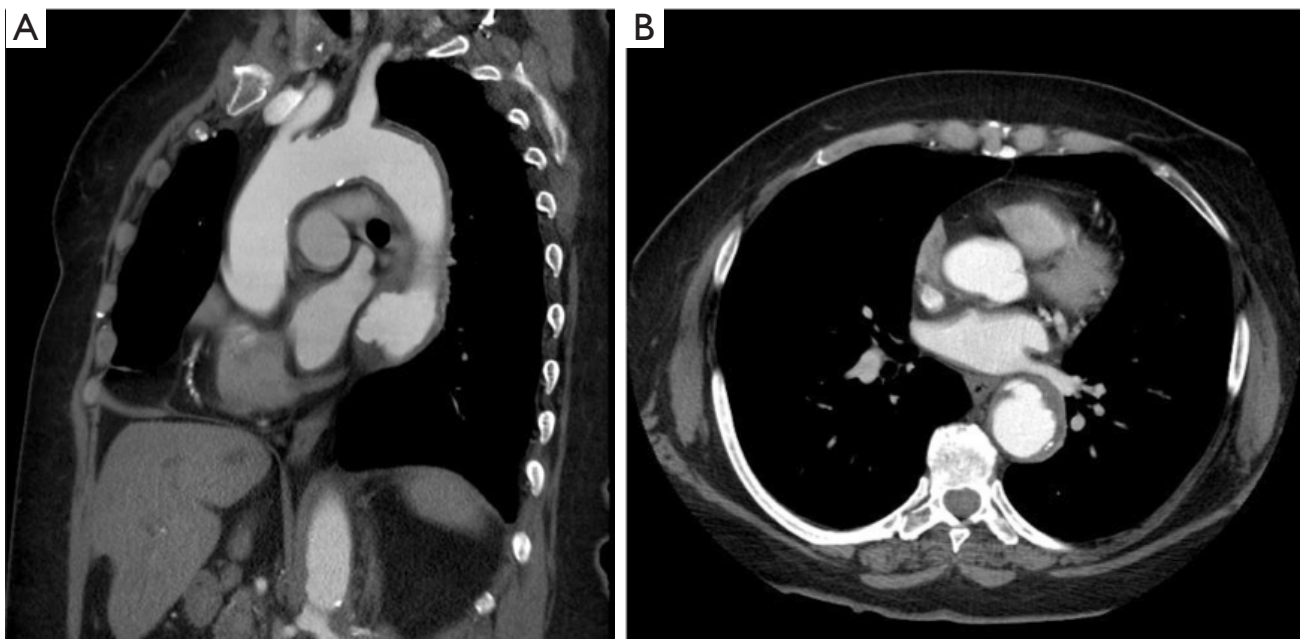

Figure 1 Retro-TAIMH that originates in the descending aorta with retrograde extension into the arch, on a sagittal view (A) and axial view (B). TAIMH, type A intramural hematoma.

\section{Etiology and clinical presentation}

The etiology of IMH has not been completely elucidated. It has been suggested that IMH originates from ruptured vasa vasorum in weak areas of the medial layer of the aortic wall, triggering a tear into the aortic lumen $(7,8)$. Another proposed concept is that IMH originates from small intimal tears followed by thrombosis of these tears, making the tears difficult to detect on imaging studies $(8,9)$. Both concepts are likely to be induced by increased wall stresses on already weakened tissue. Several potential clinical risk factors have been identified for IMH, such as higher age, large aortic diameter and increased aortic wall thickness $(6,10,11)$.

The clinical presentation of IMH patients is quite comparable to those suffering from AD. The vast majority present with acute chest pain and may show hemodynamic instability (7). However, the Houston group showed that age over 65 years, female gender, Marfan syndrome and retrograde dissection are all significantly correlated to TAIMH when compared to type A aortic dissection (TAAD) (12). Moreover, IMH patients show fewer distal malperfusion syndromes, such as mesenteric or limb ischemia, than AD patients, as there is no intimal flap disrupting aortic flow $(6,11,12)$. In addition, cardiac tamponade is more frequently found in TAAD than in TAIMH as result of $\mathrm{AD}$ rupture into the pericardium $(6,13)$.

\section{Diagnostic imaging}

Modern imaging has led to a better understanding of aortic injuries. It has been shown that $\mathrm{IMH}$ is relatively common in patients with suspected AD (10\% to $30 \%)$ (14-18). High quality CT and MRI imaging show that IMH and AD may be present at the same time in different levels of the aorta (Figure 2). Aortic dissection presenting with a thrombosed false lumen can resemble an IMH as entry tears are often no longer visible. In order to differentiate between these conditions, cross-sectional imaging is considered the gold standard. Importantly, the close relationship between an IMH and the adventitia may lead to aortic rupture (19). It is therefore vital to distinguish pleural effusion, usually a benign sign, from a peri-aortic hematoma, frequently a sign of (impending) aortic rupture. The presence of periaortic hematoma is suggestive of $\mathrm{IMH}$ rather than $\mathrm{AD}$, as it is comparatively more commonly associated with IMH. Furthermore, high quality imaging can expose small intimal lesions in the inner curvature of the aortic arch, which are more common in IMH than in AD. Such signs may support the process of making a diagnosis, as well as the planning of thoracic endovascular aortic repair (TEVAR) (20). Further radiological differences between $\mathrm{IMH}$ and $\mathrm{AD}$ involve aortic wall thickening and the shape of hematomas. For IMH, axial imaging reveals thickening of the aortic wall greater than $0.5 \mathrm{~cm}$ in an eccentric or concentric pattern, with a linear intraluminal filling defect as a distinguished feature (Figure 3). On the contrary, thrombosed false lumen in acute $\mathrm{AD}$ shows curvilinear intramural clots, often missing a well-defined outer wall because of mediastinal hematoma and pleural effusions (14). As for aortic diameters, no clear 

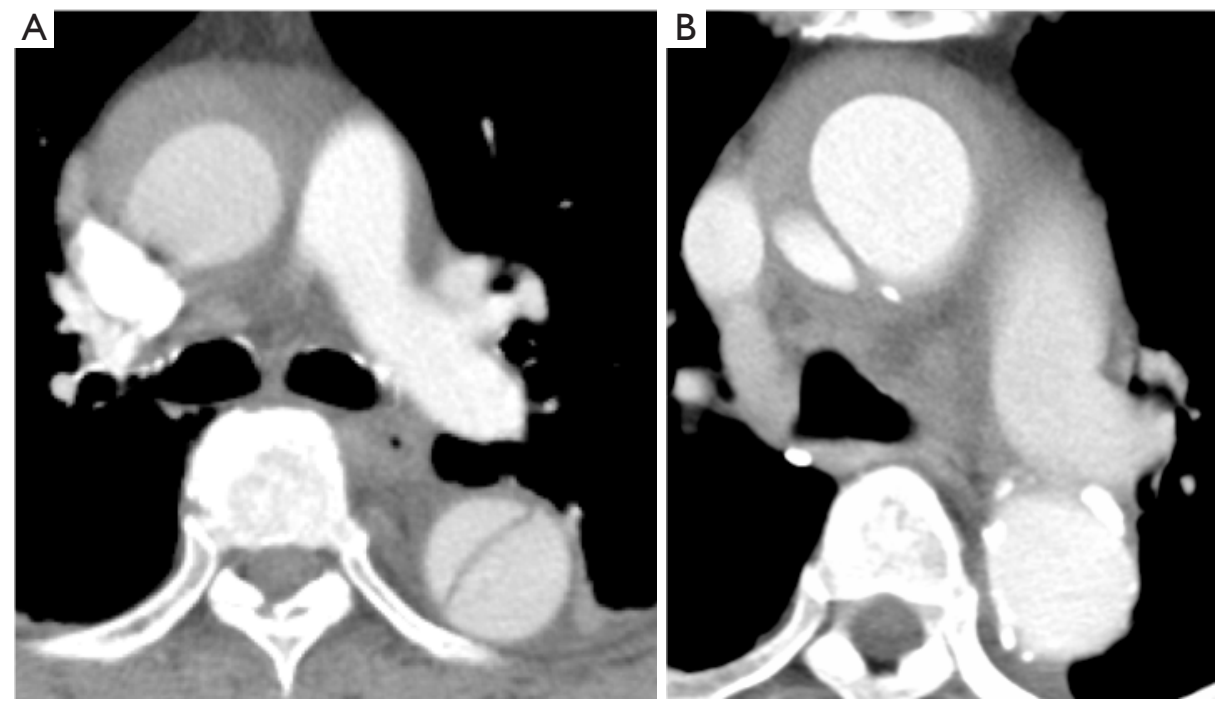

Figure 2 Retro-IMH that originates in the descending aorta (A) with retrograde extension into the ascending (B). IMH, intramural hematoma.
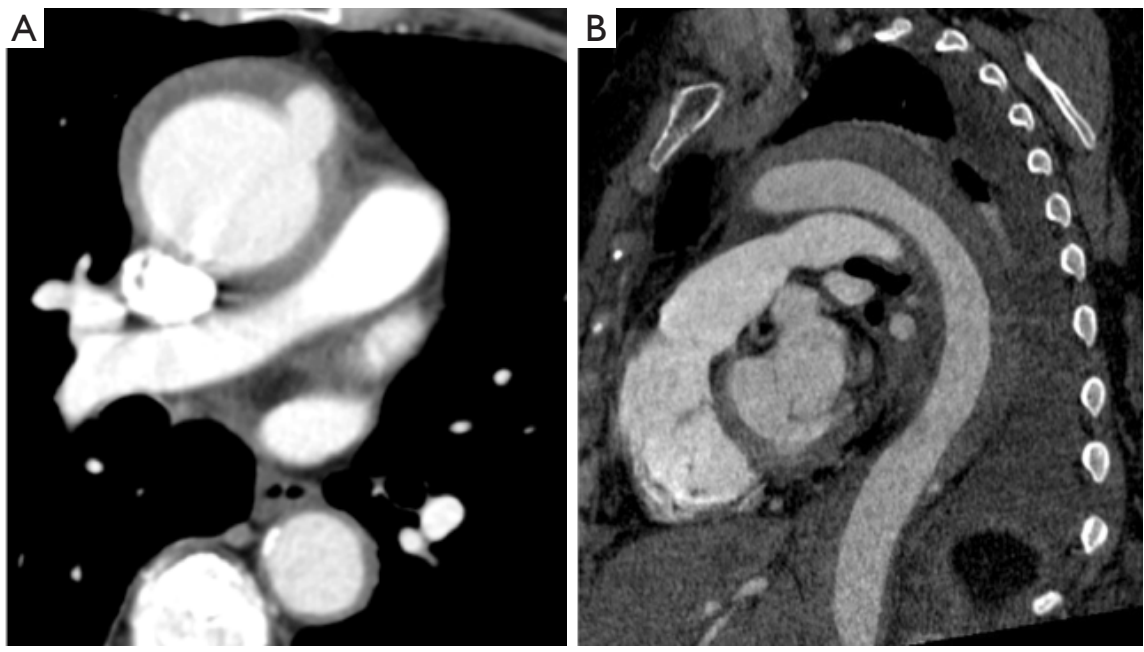

Figure 3 Axial view of ascending aortic wall thickening suggesting IMH (A). Sagittal view of IMH in the descending aorta with aortic wall thickening and linear intraluminal filling defect (B). IMH, intramural hematoma.

differences were found between patients with $\mathrm{IMH}$ or $\mathrm{AD}$, with an average of $5 \mathrm{~cm}$ for the ascending aorta and $4 \mathrm{~cm}$ in the descending aorta $(6,21)$.

\section{Prognosis}

The prognosis of acute IMH is comparable to that of acute AD. While it was formerly thought that IMH would be less dangerous, several clinical studies have underlined that early and long-term mortality of IMH do not differ from $\mathrm{AD}(6,11,13)$. In-hospital mortality varies between $12-26 \%$ for type $\mathrm{A} I M H$ and $\mathrm{AD}$, and from $5 \%$ to $11 \%$ for TBIMH and $\mathrm{AD}(6,11,21)$. Similar to TAAD, IMH involving the ascending aorta is a lethal condition and is an indication for expeditious surgery because of the risk of cardiac tamponade, rupture or compression of the coronary ostia $(6,8,10,11)$. In particular, IMH concomitant with PAU is associated with an increased risk of expansion and rupture 


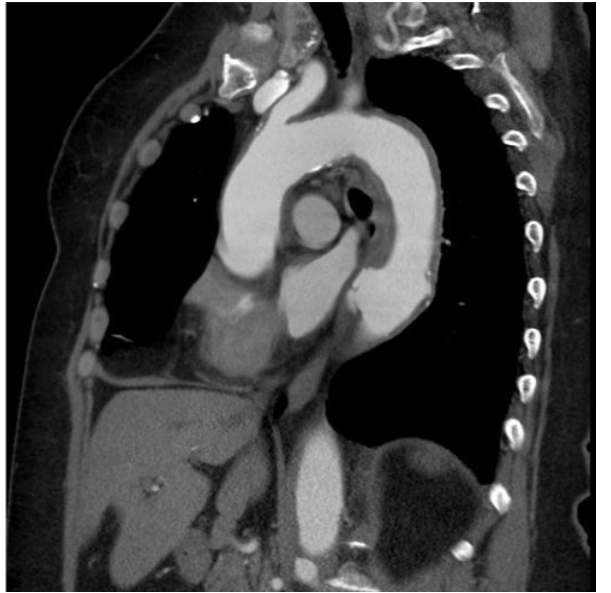

Figure $4 \mathrm{IMH}$ concomitant with a penetrating aortic ulcer. IMH, intramural hematoma.

$(14,22)$ (Figure 4). For IMH alone, even though $34 \%$ of patients will show regression, $16 \%$ to $47 \%$ of patients will progress to development of $\mathrm{AD}(8,10,22,23)$ and $20 \%$ to $45 \%$ will develop an aortic rupture (8). The best predictor of IMH regression without complications is a normal aortic diameter in the acute phase (19). With regards to adverse aortic remodelling, Evangelista et al. reported that amongst $68 \mathrm{IMH}$ patients, $22 \%$ developed a fusiform aneurysm, $8 \%$ a saccular aneurysm and $24 \%$ a pseudoaneurysm (over a mean time of 45 months) (23). It is therefore important to monitor all IMH patients closely.

\section{Management trends and recommendations}

\section{Medical}

Optimal management of TAIMH remains debatable. Most Western sites opt for emergent surgical aortic repair for unstable patients. Nonetheless, several Asian sites have reported promising results with initial medical treatment in TAIMH patients $(2,24)$. It is important to note that such centres report markedly higher incidences of TAIMH in acute aortic syndrome patients than Western sites. It is still unclear if this is caused by different definitions or by a truly different etiology. Currently, in most IRAD centres, patients who present with TAIMH but are clinically stable at presentation are typically first managed medically, in anticipation of sub-acute surgical repair $(7,12)$.

In general, IMH located in the aortic arch or descending aorta are less likely to be associated with adverse outcomes than TAIMH, and therefore best medical therapy may suffice (10). Primary management of patients presenting with uncomplicated IMH consists of medical therapy and intensive monitoring $(6,11,18)$. Medical management includes urgent blood pressure normalization and left ventricular ejection fraction reduction, as they are the main determinants of dissection extension and rupture. $\beta$-blockers have been shown to decrease mortality by $67 \%$ to $95 \%$ (22) and should be given at highest tolerated doses. Calcium channel blockers are considered the alternative medication of choice. To normalize hypertension caused by stimulation of adrenergic receptors, adequate analgesic therapy should be initiated, preferably with morphine sulphate $(10,11,14,18,22,25)$. For TBIMH patients, refractory chest pain, evidence of increasing size of the hematoma, aortic rupture and progressive pleural effusion are indications for endovascular or surgical treatment (22).

\section{Thoracic endovascular repair}

Endovascular repair is indicated in symptomatic/complicated TBIMH patients due to the risk of rupture $(14,25,26)$ (Figure 5) and is associated with lower perioperative morbidity and mortality than open repair (20,27-29). The focal character of the aortic lesion makes TBIMH patients suitable candidates for endovascular treatment $(11,30)$. Although the literature provides no convincing guidelines for IMH treatment, it seems reasonable that it is similar to treatment of type $\mathrm{B} A \mathrm{D}$ in corresponding segments of the aorta $(31,32)$. Currently, TEVAR may be indicated in patients with progression of IMH towards overt dissection or rupture (20,31). However, an important risk of TEVAR in extended IMH is that the endograft may tear through the intimal surface into underlying thrombosed false lumen. Thus, the endograft should be anchored in the noninvolved wall above and below the intimal defect (32). A recent study by Kuo et al. showed that the presence of IMH at the proximal landing of TEVAR seemed to increase the risk of retrograde TAAD (6.7\% vs. 0\%) (33). Although this finding was not statistically significantly, it suggests that a 2-cm IMH-free landing zone should be obtained, even if this means that an arch debranching is necessary. In patients presenting with retro-AIMH, the risk of neurological or cardiac complications is high, and therefore an open surgical or hybrid approach is most appropriate $(11,34)$ (Figure 6). The risk of landing in an unsuitable proximal landing zone (i.e., development of a retrograde dissection), which is the main concern of endovascular repair in retro-IMH, might be of less relevance in retrograde TAAD, since the 
A

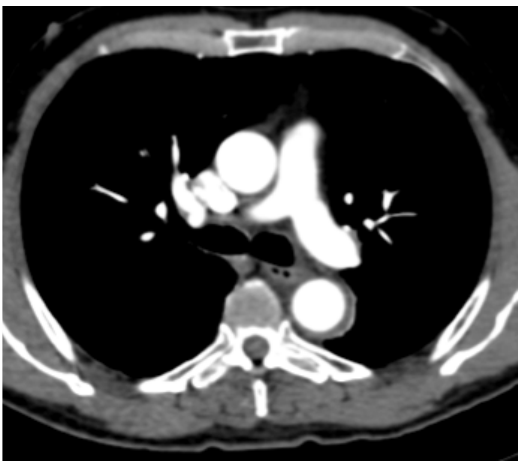

Day 5

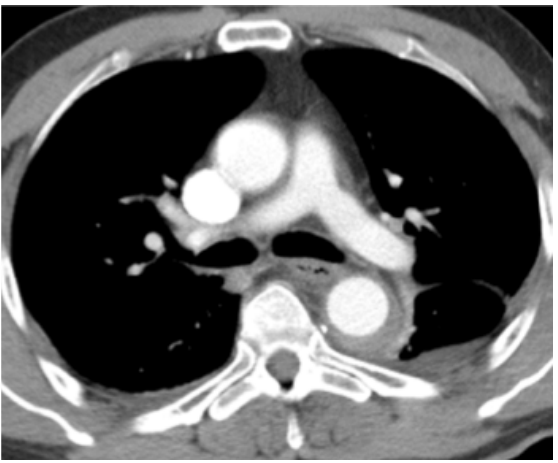

Day 10

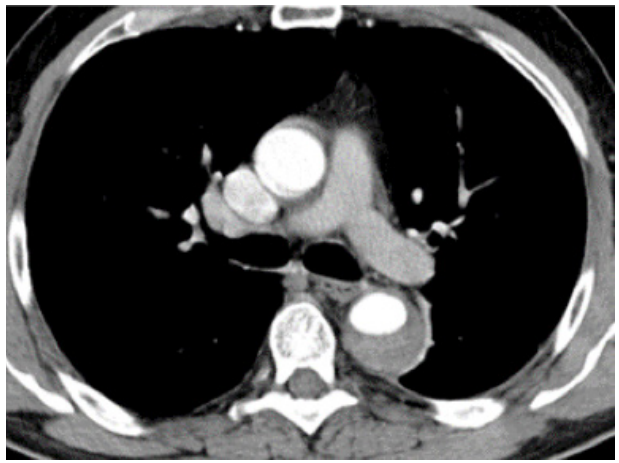

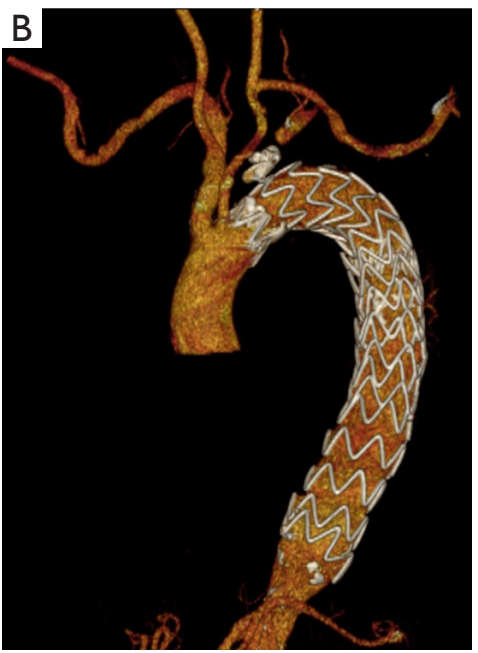

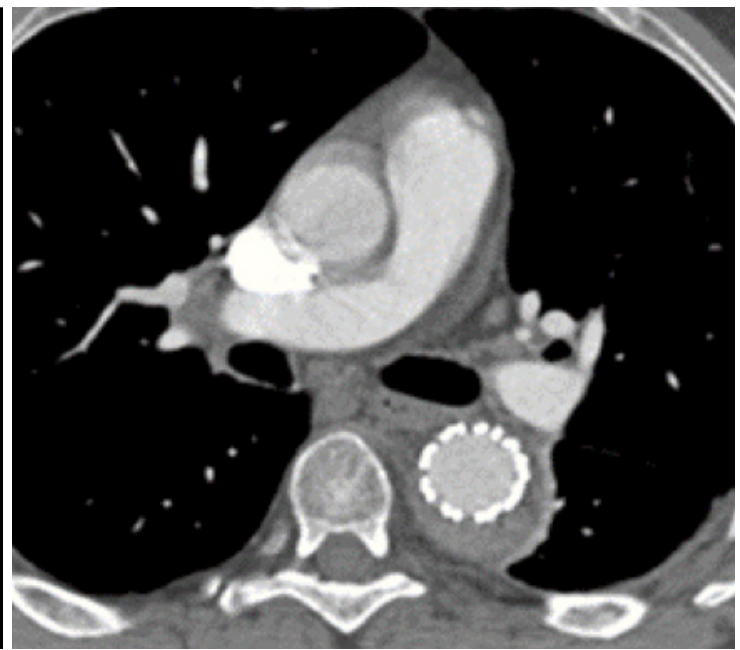

Figure $5 \mathrm{IMH}$ with crescentic thickness evolving over time, with recurrent pain at day 10 (A). TEVAR in Z2 with left carotid-subclavian bypass and plug at the LSA origin, and the axial view (B). IMH, intramural hematoma; TEVAR, thoracic endovascular aortic repair.

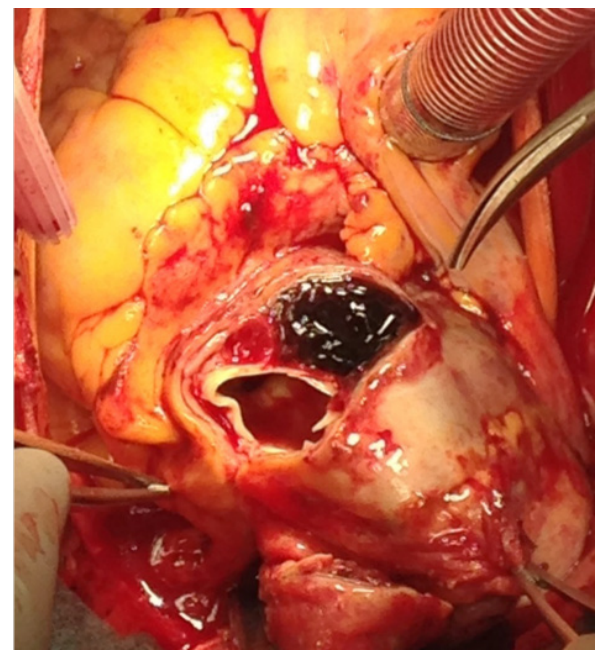

Figure 6 Ascending aortic intramural hematoma. retrograde dissection is already there. In a still unpublished work, Omura et al. describe the use of TEVAR in landing zones 1 to 4 for a small subgroup of patients with retrograde TAAD and an entry tear in the descending aorta (35). These 8 patients were considered to be at prohibitive surgical risk, but the achieved outcome was very good: 30 -day mortality was $0 \%$ and, interestingly, postoperative CT scans showed complete thrombosis and reduction in size of the false lumen in the ascending aorta in all patients (Figure 7). In addition, some small case series on retroTAIMH have recently reported acceptable outcomes with endovascular repair $(36,37)$.

\section{Surgical}

Surgical aortic repair remains the gold standard for patients 

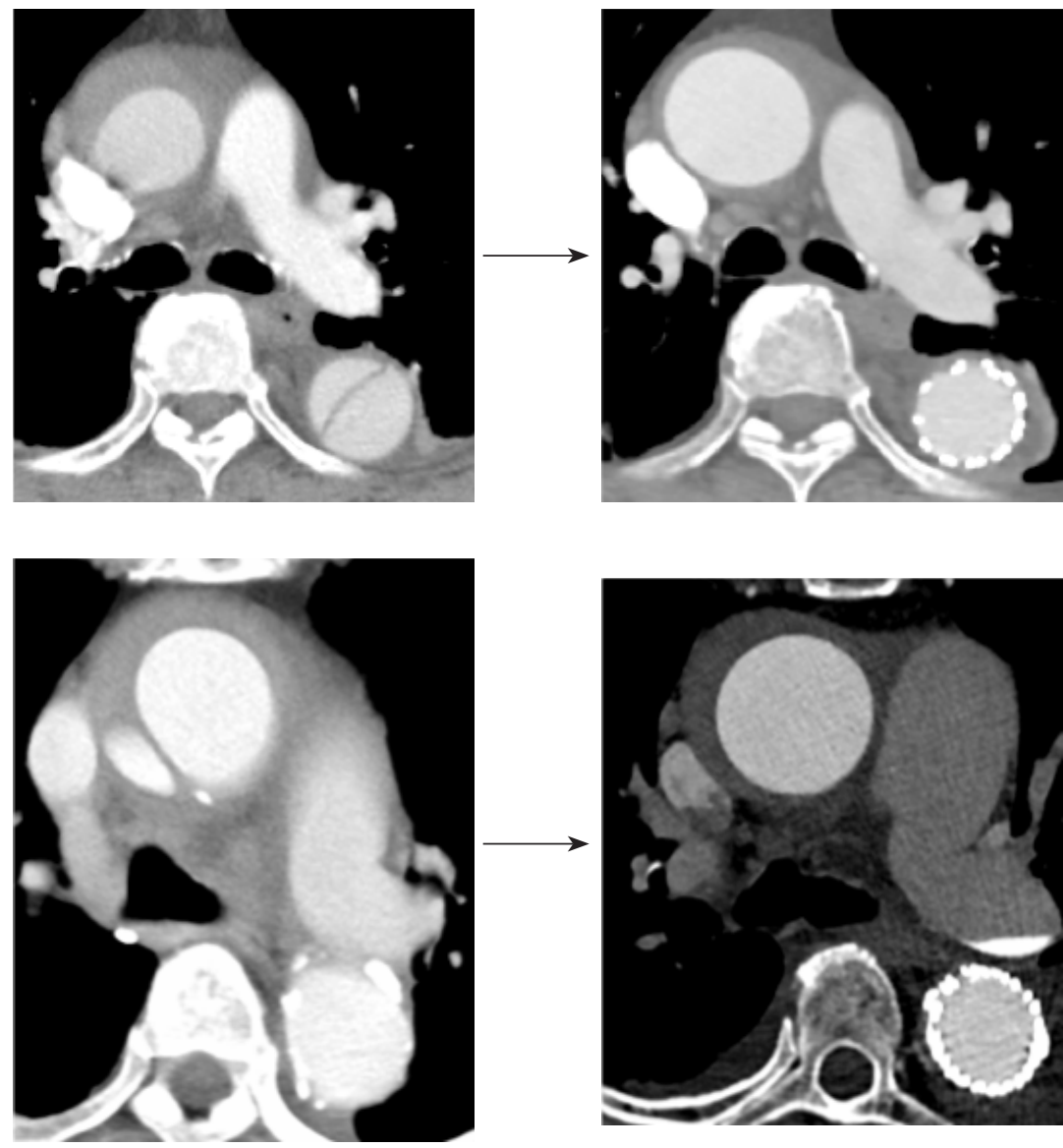

Pre-operative

Post-operative

Figure 7 Aortic remodeling following thoracic endovascular repair for retrograde type A aortic dissection.

presenting with complicated TAIMH (hemodynamic instability, persistent pain, or signs of rupture) $(7,31,32)$. The surgical technique in TAIMH consists of ascending aortic repair to prevent aortic rupture and proximal extension of the IMH. Although the aortic wall adjacent to the IMH might not be visibly pathological, the use of felt to reinforce the anastomoses is indispensable, and bioglue is often added as well. The distal extent of repair is determined by the diameter of the aortic arch. Enlargement of the $\operatorname{arch}>5 \mathrm{~cm}$ is not uncommon, especially in cases of type B dissection with retro-TAIMH, and concomitant arch repair may be required (12). Some centers even use a threshold of $4 \mathrm{~cm}$ (38). If this is not the case, a partial arch replacement with open distal anastomosis may suffice. Use of deep hypothermic circulatory arrest with selective cerebral perfusion, in combination with intraoperative cerebral monitoring with transcranial Doppler and/or EEG, is generally required.
The proximal extent of the repair is determined by involvement of the aortic root and aortic valve. As these are less commonly involved in TAIMH than in TAAD, aortic root replacement or aortic valve resuspension are not as often required in TAIMH (15\%) as in TAAD (36\%) (6). Therefore, surgical procedure times for TAIMH are significantly shorter than for TAAD. However, outcomes did not differ for stroke (13\% and $7 \%$ respectively) and mortality (12-26\% and $16-26 \%$ respectively $(6,12)$. In the IRAD group, 24 patients had the most proximal extension of IMH in the arch. Of these, 16 were medically managed, 4 were surgically managed, 2 were managed with endovascular therapy, and 2 by a hybrid approach $(6,39)$.

A hybrid approach can be considered, especially in retroTAIMH patients. There are two main options for hybrid repair. The first is (supracoronary) ascending and partial arch replacement with debranching of the brachiocephalic and left common carotid artery, creating a safe landing 

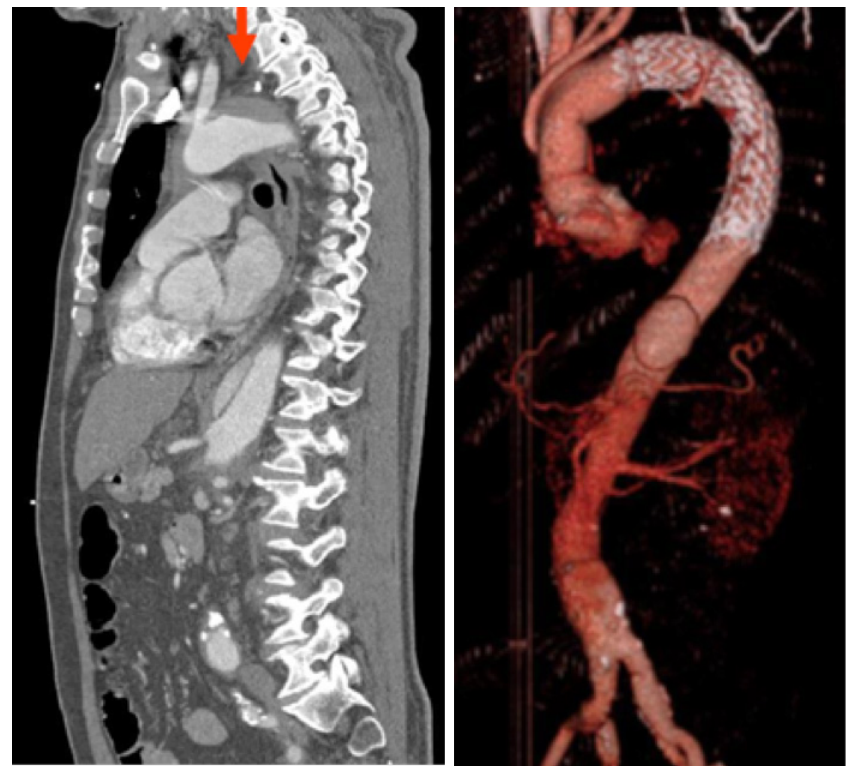

Figure 8 CT-imaging of a patient with retro-TAIMH in the arch. The red arrow shows the thicker section of the retro IMH located at the origin of the left common carotid artery. The patient was managed with ascending-arch repair and frozen elephant trunk. TAIMH, type A intramural hematoma.

zone for subsequent TEVAR, which is done to complete the procedure. This option allows relatively good exposure and controlled creation of the distal anastomosis, and might be preferable in the acute setting. A downside is the deliberate overstenting of the left subclavian artery, as it is often unknown whether a patient has a complete circle of Willis. The second option is ascending and total arch replacement with a frozen elephant trunk (Figure 8) with additional TEVAR if necessary, in a single stage procedure. As experience with hybrid techniques is increasing, such extensive repairs are gaining popularity for the acute setting, since they are thought to offer more favorable results on aortic remodeling of the thoracoabdominal aorta. However, there are currently no reports describing the surgical results specifically for retro-TAIMH. For TAAD, a pooled analysis of published results of hybrid techniques showed a 30-day mortality of $5-12 \%$ (40). It should be noted that these results are based on retrospective analyses, so there is a potential publication bias.

Optimal management of uncomplicated TAIMH is a matter of debate. Some authors state that these patients may be managed medically, and have reported promising results $(4,41)$. However, about $25 \%$ of these patients still need urgent surgical repair (41). In another series, $43 \%$ of patients who were initially managed medically needed surgical repair within 1 year of presentation (42). In the IRAD group, of 10 patients with TAIMH managed medically, 4 patients died in-hospital (3 because of rupture and 1 due to aortic dissection with mesenteric ischemia). Therefore, patients with TAIMH who are managed medically should be monitored closely $(4,41)$.

\section{Follow up}

A five-year follow-up for both IMH type A and type B is advised (43). IRAD investigators believe life-long medical therapy for strict blood pressure regulation is indicated for all patients $(11,44)$. In addition, it has been reported that aortic enlargement for TBIMH during follow-up was significantly less common compared to type $\mathrm{B} A D$ patients (39\% vs. 61\%; $\mathrm{P}=0.034)$ (11).

\section{Conclusions}

Retro-TAIMH is an acute aortic syndrome and should be managed as such. The recommended treatment strategy is open surgery for treating ascending or arch involvement, and TEVAR/medical, based on a complication-specific approach, for those with only descending localization. For patients in whom retro-TAIMH is associated with an acute type $\mathrm{B}$ dissection presenting with a proximal entry tear located into the descending aorta, TEVAR is an option for treatment.

\section{Acknowledgments}

None.

\section{Footnote}

Conflicts of Interest: The authors have no conflicts of interest to declare.

\section{References}

1. Estrera A, Miller C 3rd, Lee TY, et al. Acute type A intramural hematoma: analysis of current management strategy. Circulation 2009;120:S287-91.

2. Kitai T, Kaji S, Yamamuro A, et al. Clinical outcomes of medical therapy and timely operation in initially diagnosed type a aortic intramural hematoma: a 20 -year experience. 
Circulation 2009;120:S292-8.

3. Pelzel JM, Braverman AC, Hirsch AT, et al. International heterogeneity in diagnostic frequency and clinical outcomes of ascending aortic intramural hematoma. J Am Soc Echocardiogr 2007;20:1260-8.

4. Song JK, Kim HS, Song JM, et al. Outcomes of medically treated patients with aortic intramural hematoma. Am J Med 2002;113:181-7.

5. Uchida K, Imoto K, Takahashi M, et al. Pathologic characteristics and surgical indications of superacute type A intramural hematoma. Ann Thorac Surg 2005;79:1518-21.

6. Harris KM, Braverman AC, Eagle KA, et al. Acute aortic intramural hematoma: an analysis from the International Registry of Acute Aortic Dissection. Circulation 2012;126:S91-6.

7. Mussa FF, Horton JD, Moridzadeh R, et al. Acute Aortic Dissection and Intramural Hematoma: A Systematic Review. JAMA 2016;316:754-63.

8. Nienaber CA, Eagle KA. Aortic dissection: new frontiers in diagnosis and management: Part I: from etiology to diagnostic strategies. Circulation 2003;108:628-35.

9. Nienaber CA, Sievers HH. Intramural hematoma in acute aortic syndrome: more than one variant of dissection? Circulation 2002;106:284-5.

10. Evangelista A, Mukherjee D, Mehta RH, et al. Acute intramural hematoma of the aorta: a mystery in evolution. Circulation 2005;111:1063-70.

11. Tolenaar JL, Harris KM, Upchurch GR Jr, et al. The differences and similarities between intramural hematoma of the descending aorta and acute type B dissection. J Vasc Surg 2013;58:1498-504.

12. Sandhu HK, Tanaka A, Charlton-Ouw KM, et al. Outcomes and management of type A intramural hematoma. Ann Cardiothorac Surg 2016;5:317-27.

13. De Martino RR, Brooke BS, Robinson $W$, et al. Designation as "unfit for open repair" is associated with poor outcomes after endovascular aortic aneurysm repair. Circ Cardiovasc Qual Outcomes 2013;6:575-81.

14. Ganaha F, Miller DC, Sugimoto K, et al. Prognosis of aortic intramural hematoma with and without penetrating atherosclerotic ulcer: a clinical and radiological analysis. Circulation 2002;106:342-8.

15. Nienaber CA, von Kodolitsch Y, Petersen B, et al. Intramural hemorrhage of the thoracic aorta. Diagnostic and therapeutic implications. Circulation 1995;92:1465-72.

16. O'Gara PT, DeSanctis RW. Acute aortic dissection and its variants. Toward a common diagnostic and therapeutic approach. Circulation 1995;92:1376-8.
17. Shimizu H, Yoshino H, Udagawa H, et al. Prognosis of aortic intramural hemorrhage compared with classic aortic dissection. Am J Cardiol 2000;85:792-5, A10.

18. von Kodolitsch Y, Csosz SK, Koschyk DH, et al. Intramural hematoma of the aorta: predictors of progression to dissection and rupture. Circulation 2003;107:1158-63.

19. Evangelista A, Dominguez R, Sebastia C, et al. Prognostic value of clinical and morphologic findings in short-term evolution of aortic intramural haematoma. Therapeutic implications. Eur Heart J 2004;25:81-7.

20. Eggebrecht H, Plicht B, Kahlert P, et al. Intramural hematoma and penetrating ulcers: indications to endovascular treatment. Eur J Vasc Endovasc Surg 2009;38:659-65.

21. Estrera AL, Sandhu H, Afifi RO, et al. Open repair of chronic complicated type B aortic dissection using the open distal technique. Ann Cardiothorac Surg 2014;3:375-84.

22. Ince $H$ and Nienaber CA. Diagnosis and management of patients with aortic dissection. Heart 2007;93:266-70.

23. Evangelista A, Dominguez R, Sebastia C, et al. Long-term follow-up of aortic intramural hematoma: predictors of outcome. Circulation 2003;108:583-9.

24. Song JK, Yim JH, Ahn JM, et al. Outcomes of patients with acute type a aortic intramural hematoma. Circulation 2009;120:2046-52.

25. Nathan DP, Boonn W, Lai E, et al. Presentation, complications, and natural history of penetrating atherosclerotic ulcer disease. J Vasc Surg 2012;55:10-5.

26. Nienaber CA, Fattori R, Lund G, et al. Nonsurgical reconstruction of thoracic aortic dissection by stent-graft placement. N Engl J Med 1999;340:1539-45.

27. Clough RE, Mani K, Lyons OT, et al. Endovascular treatment of acute aortic syndrome. J Vasc Surg 2011;54:1580-7.

28. Geisbüsch P, Kotelis D, Weber TF, et al. Early and midterm results after endovascular stent graft repair of penetrating aortic ulcers. J Vasc Surg 2008;48:1361-8.

29. Leurs LJ, Bell R, Degrieck Y, et al. Endovascular treatment of thoracic aortic diseases: combined experience from the EUROSTAR and United Kingdom Thoracic Endograft registries. J Vasc Surg 2004;40:670-9; discussion 679-80.

30. Sundt TM. Intramural hematoma and penetrating atherosclerotic ulcer of the aorta. Ann Thorac Surg 2007;83:S835-41; discussion S846-50.

31. Erbel R, Aboyans V, Boileau C, et al. 2014 ESC 
Guidelines on the diagnosis and treatment of aortic diseases: Document covering acute and chronic aortic diseases of the thoracic and abdominal aorta of the adult. The Task Force for the Diagnosis and Treatment of Aortic Diseases of the European Society of Cardiology (ESC). Eur Heart J 2014;35:2873-926.

32. Hiratzka LF, Bakris GL, Beckman JA, et al. 2010 ACCF/AHA/AATS/ACR/ASA/SCA/SCAI/SIR/STS/ SVM guidelines for the diagnosis and management of patients with Thoracic Aortic Disease: a report of the American College of Cardiology Foundation/American Heart Association Task Force on Practice Guidelines, American Association for Thoracic Surgery, American College of Radiology, American Stroke Association, Society of Cardiovascular Anesthesiologists, Society for Cardiovascular Angiography and Interventions, Society of Interventional Radiology, Society of Thoracic Surgeons, and Society for Vascular Medicine. Circulation 2010;121:e266-369.

33. Kuo EC, Veranyan N, Johnson CE, et al. Impact of proximal seal zone length and intramural hematoma on clinical outcomes and aortic remodeling after thoracic endovascular aortic repair for aortic dissections. J Vasc Surg 2019;69:987-95.

34. Bavaria JE, Brinkman WT, Hughes GC, et al. Outcomes of Thoracic Endovascular Aortic Repair in Acute Type B Aortic Dissection: Results From the Valiant United States Investigational Device Exemption Study. Ann Thorac Surg 2015;100:802-8; discussion 808-9.

35. Matsuda H. Thoracic endovascular repair for retrograde acute type A aortic dissection as an alternative choice. Personal communication, GICS 2019, Gwangju, South Korea.

36. Haenen FWN, Van Der Weijde E, Vos JA, et al.

Cite this article as: Nauta F, de Beaufort H, Mussa FF, De Vincentiis C, Omura A, Matsuda H, Trimarchi S. Management of retrograde type A IMH with acute arch tear/type B dissection. Ann Cardiothorac Surg 2019;8(5):531-539. doi: 10.21037/acs.2019.08.05
Retrograde Type A Intramural Hematoma Treated Endovascularly in Two Cases. Ann Vasc Surg 2019;59:312. e15-312.e18.

37. Vo AT, Le KM, Nguyen TT, et al. Acute retrograde type A intramural hematoma treated with an endovascular approach. Asian Cardiovasc Thorac Ann 2018;26:308-10.

38. Yang B, Norton EL, Shih T, et al. Late outcomes of strategic arch resection in acute type A aortic dissection. J Thorac Cardiovasc Surg 2019;157:1313-1321.e2.

39. Nauta FJ, Tolenaar JL, Patel HJ, et al. Impact of Retrograde Arch Extension in Acute Type B Aortic Dissection on Management and Outcomes. Ann Thorac Surg 2016;102:2036-43.

40. Smith HN, Boodhwani M, Ouzounian M, et al. Classification and outcomes of extended arch repair for acute Type A aortic dissection: a systematic review and meta-analysis. Interact Cardiovasc Thorac Surg 2017;24:450-9.

41. Motoyoshi N, Moizumi Y, Komatsu T, et al. Intramural hematoma and dissection involving ascending aorta: the clinical features and prognosis. Eur J Cardiothorac Surg 2003;24:237-42; discussion 242.

42. Chou AS, Ziganshin BA, Charilaou P, et al. Long-term behavior of aortic intramural hematomas and penetrating ulcers. J Thorac Cardiovasc Surg 2016;151:361-72, 373.e1.

43. Moizumi Y, Komatsu T, Motoyoshi N, et al. Clinical features and long-term outcome of type A and type B intramural hematoma of the aorta. J Thorac Cardiovasc Surg 2004;127:421-7.

44. Nauta FJH, Kim JB, Patel HJ, et al. Early Outcomes of Acute Retrograde Dissection From the International Registry of Acute Aortic Dissection. Semin Thorac Cardiovasc Surg 2017;29:150-9. 